\title{
Implementing Epic Beaker Laboratory Information System for Diagnostics in Anatomic Pathology
}

\author{
Consolato M Sergi $\mathbb{D}^{1,2}$ \\ 'Anatomic Pathology Division, Children's Hospital of Eastern Ontario, University of Ottawa, Ottawa, ON, Canada; ${ }^{2}$ Deparment of Laboratory \\ Medicine and Pathology, University of Alberta, Edmonton, AB, Canada \\ Correspondence: Consolato M Sergi, Tel + I 6I3-737-7600 x 2427, Fax + I 6I3-738-4837, Email csergi@cheo.on.ca
}

\begin{abstract}
Medicine is expeditiously evolving, and the number of diagnostic opportunities has increased exponentially in the last decade. Electronic medical records (EMRs) have been welcomed in most institutions worldwide following an early period of suspicious behavior. Unfortunately, several cracks dictated the initial approach to hospital systems and leadership incompetency. However, the pathway for a successful decade of EMRs is paved. This narrative review illustrates some principles implementing Epic Beaker software for anatomic pathology in academic medical institutions. Implementing such software improves the diagnostic approach in the division of anatomic pathology because the pathologists can directly access an enormous amount of clinical and radiological information now at their front desk using extremely versatile windows.
\end{abstract}

Keywords: diagnostics, laboratory information system, Epic, Beaker, quality assurance

\section{Introduction}

Medicine is rapidly evolving, and the number of diagnostic opportunities and challenges has increased exponentially in the last decade. Electronic medical records (EMRs), also known as electronic health records (EHR) have been welcomed in most institutions worldwide following an early period of suspicious behavior. Several cracks dictated the initial approach to hospital systems and leadership incompetency. However, the pathway for a successful decade of EMR is paved. The future of medicine is constellated by increasing bureaucracy and administrative duties for physicians, but a functional and versatile laboratory information system (LIS) will help the physician enormously. Thus, EMR may represent relief for the administration because it should reduce the desk work and leave the physician to dedicate more time for the patients. However, there is a learning curve for several EMR software that has to be considered, and reports of incidents or near-misses are crucial for quality assurance in healthcare. ${ }^{1}$

Digitalization in medical imaging departments is truly experienced in several healthcare institutions worldwide, despite hits and controversies. The disputes of filing properly and in a confidential way have been superbly solved in numerous departments. However, the making digital of routine light microscopic slides has shown an initial slow pace with more sprints in the last couple of years during the COVID-19 pandemic. The application of specific programs for external quality assurance is currently setup for many pathologists and pathology offices, and programs targeting continuing medical education are becoming mandatory and opting for the use of virtual microscopy in numerous institutions. The leaving of traditional light microscopic slides using glass for the daily routine diagnostics is approaching many laboratory medicine departments and pathology departments, but the pace is different between provinces and provinces as well as states and states. Since 1986, telepathology services to transfer images of anatomic pathology between separate settings have helped countless patients universally, including the Canada based University of Alberta. The idea of specialist (pathologist) recertification or revalidation is a primary milestone. Challenges, such as high bandwidth requirements, electronic platforms, and the central processing units' and operating systems' stability, have been aimed and are enhancing immensely. The safe encoding of digital images may become an absolute prerequisite for the official recognition of future laboratory services. ${ }^{2-4}$ 
Beaker is a system operating as an LIS offered in the United States and Canada by Epic Systems Corporation (Verona, WI, United States). This LIS can be set up in two independent modules. Beaker Anatomic Pathology (also known as "Beaker AP") and Beaker Clinical Pathology (also known as "Beaker CP") represent the two modules. Beaker is a fairly new LIS within the Epic suite, which offers both LIS and EMR. Provided that Epic is a United States popular EMR, clinical laboratories appraising LIS options might ponder Beaker a such an option, specifically if their institution at this time operates the Epic as an EMR solution. In addition, Beaker is commonly incorporated in enterprise licenses for user of the Epic software. This narrative review illustrates some principles implementing Epic Beaker software for anatomic pathology in academic medical institutions. Implementing such software will improve the diagnostic approach because the pathologists will directly access an enormous amount of clinical and radiological information now at their front desk.

\section{Materials and Methods}

Articles involving Epic Beaker's implementation have been identified in PubMed, and suggestions and comments from blogs and online resources have been consulted. ${ }^{5-9}$ Five articles have been examined, and systems and surveys evaluated. In evaluating the manuscripts, the LIS execution impacted all the AP laboratories, including cytopathology, surgical pathology, and autopsy. Also, some CP testing was supposed to suit better with the Beaker AP module. Thus, it was set aside for implementing that module.

\section{Results and Discussion}

One of the most intriguing aspects in Epic Beaker is the tissue-based workflow, which we depicted in Figure 1. All Epic applications use a single shared list, which is called the Specimen Source Dictionary ORD325. Blau et al

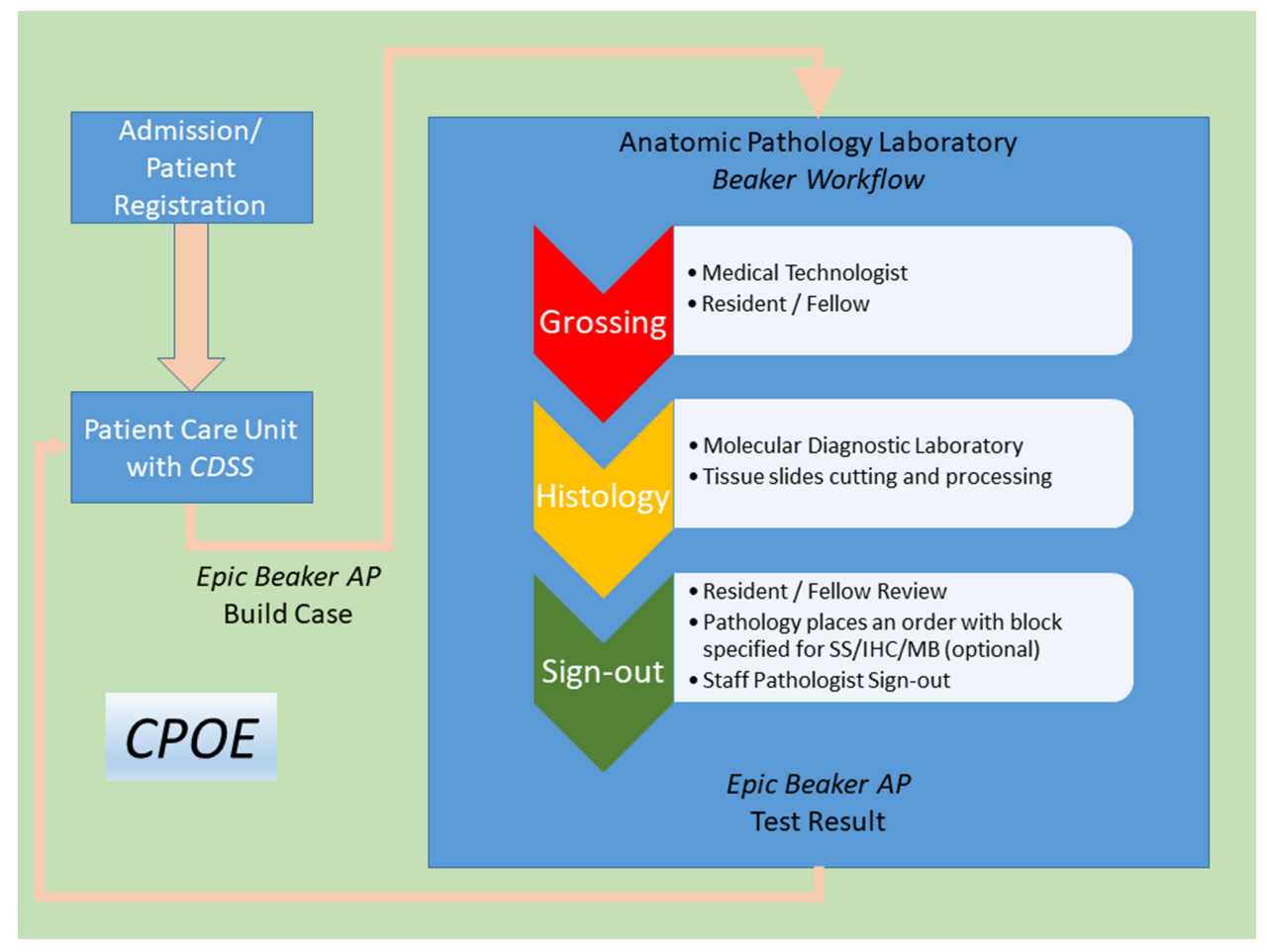

Figure I Tissue-based workflow in Epic Beaker. Following admission or patient registration, patient care unit will lead the process with the Clinical Decision Support System (CDSS). The CDSS helps physicians to choose evidence-based decisions, regarding patients' needs and this is the major rational task, which targets to be implemented worldwide. In a computerized provider order entry (CPOE) environment, the anatomic pathology Beaker Workflow includes grossing, histology, and sign-out phases. Grossing involves either medical technologist or a resident/fellow. Histology involves the medical diagnostic laboratory with tissue slides cutting and processing. In the signout phase, if no additional special stains (SS), immunohistochemistry (IHC), or molecular biology tasks need to be implemented, the histopathology report can be sign-out by the staff pathologist after revision of the resident/fellow. All Epic applications use a single shared list, which is called the Specimen Source Dictionary ORD325. 
found that several parties of people employing the source list had different objectives. ${ }^{6}$ Microbiology entails that the plan be classy enough to determine which plate labels. In our opinion, it seems that it is crucial that testing procedures should be created consequently. On the other hand, the laboratory devoted to surgical pathology would like a streamlined list with reduced sources to decrease faults in the downstream choice of specimen protocols. These protocols would prompt specific cassette and slide rules as well as billing codes. This aspect may be an essential discussion with different stakeholders before implementing this LIS in an AP laboratory. Blau et $\mathrm{al}^{6}$ indicated that the specimen source thesaurus was developed with the microbiological representation in mind. Still, the source inventory needed a considerable expansion to integrate resources relevant to AP. Also, familiar sources (eg, tissue) have intersecting schemata where one resource could be associated with more than one pathology request or examination. The choice of a specific sample supply in OpTime does not permit for a default specimen protocol's automated population. Briefly, Epic OpTime is Epic's' operating room management system. It combines clinical and decision aid tools with the aim to integrate scheduling for the management of ambulatory surgical centers and hospital operating rooms. Navigator is a series of sections with the purpose to follow a specific workflow, such as an office visit or medication reconciliation. Apart from OpTime Navigator, other examples include the Visit Navigator and the Discharge Navigator. Areas not employing the OpTime Navigator need to submit orders through Order Entry or Managed Orders. This is mentioned to as the "clinic collect" method of ordering. It is essential that the users first decide on the suitable "encounter" and then select the desired order through Order Entry and Managed Orders. They do not have to choose a specific source first. Nevertheless, a substantial functional downside for utilising a Surgical Pathology template for every non-gynecologic cytology case is that each case shows that a paraffin tissue block exists, even though most of the non-gynecologic circumstances may not come up with tissue blocks. Another drawback of using the Surgical Pathology model is that no separate values can be routinely chosen, as an alternative of depending on a thesaurus of different distinct values to select from, but which do not all the time thoroughly match the final diagnosis. This issue was not resolved in Beaker AP v.2017.

The reporting module is useful to identify patients harboring a cytology case that also have had a surgery, and then, a surgical pathology case in a well-specified time. The report is crucial for cases that are contenders for the cytological/histological correlation. A form is used to file the case review and assemble all the information in Case QA of Beaker. The proposed workflow for intraoperative frozen sections was modified in one of my previous institutions (The University of Alberta, Edmonton, AB, Canada) using the Case Builder application. This flexibility is excellent in AP Beaker. A significant issue with EPIC's layout of the intraoperative frozen section function relies in that the data accessioning, grossing information, and interpretation activities are reciprocally limited. This aspect is also proper for Case Results activity. In fact, entering text data on a particular case in Case Results secures action in Case Builder and, of course, vice versa. If numerous portions on a individual issue arrive in the laboratory simultaneously, it would be not possible to promptly act on accession, gross, and interpret the intraoperative frozen section results. The group of investigators has encountered similar tissue locking issues in its initial analysis of the Beaker module of Clinical Pathology, although this aspect seems to have been solved recently. In most institutions, and in Edmonton, $\mathrm{AB}$, as well, pathologists use barcoded cassettes and a multicoloured cassette system to enable downstream visual cues for histology handling. Commonly, and in Edmonton, $\mathrm{AB}$, as well, pink cassettes are used to connote cases that necessitate immediate (STAT, Short Turn Around Testing) processing. Remarkably, all functions are multiverse, user-controlled, and universally user-friendly.

A substantial upgrade from our LIS was the capability to print slide labels in an immediate way. Laser printing labels on-demand at the microtome may require that each station is supplied with a label printer and a complete and smart operable computer workstation. During the build, most authors identified that assignment protocols had to be appointed to one of four groupings. The determinate groupings or categories are, usually, slides recuts, special stains, immunohistochemistry (IHC), molecular biology or immunofluorescence, and/or others. These categories cannot be changed, but there is the opportunity that they can be added to any additional classes in the nearest future. The "Others" category turn out to be a catch-all for several tasks, which seem to not fitting into the other conventional categories. Resting on the bench task, the technicians or histotechnologists could decide the 
suitable view to influence their work protocol. In Beaker AP's Case Preparation Work List (CPWL) there are pending tasks. However, on the CPWL screen, the operator cannot find out what picture from which they are working. To make it simpler for the technician or histotechnologist to distinguish between the different perspectives, most of the institutions allocated a color to each position to see what screen they were looking. This issue in CPWL has been brilliantly addressed in Beaker AP v.2017.

Two methods for data analytics are offered in EPIC Beaker. The first method includes ad hoc searching and report creation. The "Reporting Workbench' permits operators or managers to conduct ad hoc queries and create reports by themselves. One of the most fascinating quality of EPIC Beaker is the relatively user-friendly interface. It easily enables query generation by customers who may not be often familiar with specific software, ie, database query languages (eg, SQL, Structured Query Language). The EPIC Caché database is a version, quite specialized, of InterSystems' proprietary Caché database. EPIC Cache database is addressed for use with Epic systems and subsystems. It is a hierarchical database, which has been tailored for maximum speed, reliability, and business agility. Reporting Workbench queries take effect on the production Cache database is crucial. It underlies all the EPIC products, permitting for up-to-date results. However, provided that the questions involve the production database, it is important to realize that query run-time at most institutions, and this concerns also several Canadian hospitals, is restricted to one hour for operators mostly outside of the central department of Information and Technology. Therefore, queries associated with a query date range variable of more than 2 or even 3 weeks have a tendency to quickly to time-out before conclusion. This aspect may obviously limit the utility of "Reporting Workbench" in specific cases. This is the case when a large amount of data requires to be analyzed and processed. The "Reporting Workbench" is utilized for the laboratory dashboards to direct properly the workflow. Also, it is used to pinpoint tests that are explicitly falling behind scheduled or expected turnaround time (TAT). The second analytic route entails the Clarity database. It is an SQL-based relational database. A relational database is a collection of data items. These items harbor pre-defined relationships among them. A set of tables with columns and rows characterize the organization of data items. In a table, each row can be marked with a unique identifier ("primary key"), and rows among multiple tables can be created using foreign keys. SQL databases are clearly relational databases, and harbor a strict, predefined schema. Conversely, noSQL databases, or non-relational databases, can be graph databases, key-value pairs, document based, or wide-column stores. Clarity database is updated with new data flowing in on a regular nightly basis. In particular, queries are built with SQL. Reports are conventionally generated using Crystal reports. Creating queries involving the Clarity database is limited to several institutions' central department's of Information and Technology dedicated individuals, which are known as the reporting team. Questions submitted to the Clarity system are complete more quickly because the relational databases are obviously better than no-relational databases. The completely hierarchical database architecture of the EPIC/Beaker production database is key. This is substantially the system utilized to produce the most longterm operational reports, such as TAT, which is important for quality control and quality assurance. NoSQL have the tendency to represent a better option for applications that have more complex, constantly changing data sets, and requiring a data model, which is flexible and does not need to be immediately defined. There is one region in which these two systems of data analytics seem to break. The practice most often wished by anatomic and clinical pathologists is the capability to execute a free-text search of all pathology cases over a wide-ranging time span. It may either find a certain report or find a sequence of pieces with similar wording. The Clarity database system accumulates the large text spots. It encompass a surgical pathology report as smaller sections of text that are reorganized to produce a complete text. It must be emphasized that such a procedure may complicate the system. It creates often a free text search because the query phrase is in a text. However, if the expression is split into two of the smaller and detailed blocks of text, a simple query would necessarily miss that result and this needs to be taken into account by the pathologists.

In academia, the implementation of the Beaker AP module requires in our opinion creative thinking and necessitates a constant update of the knowledge and expertise of relational databases. It is key to conquer certain 
discrepancies between the laboratory workflow and the typical workflow developed into the Beaker LIS. First, it is crucial to find these discrepancies or incompatibilities early, through demonstrations to end-operators in the laboratory during the planning phase. Hands-on knowledge and understanding early in the build process is critical. It helps most institutions find numerous of those areas that would necessitate more work to enhance and optimize. On a number of occasions, portions of the assembly may be abandoned. In our experience and in that arising from the literature such situations start again after feedback from end-operators indicating the requirement for significant adjustments. Choices to discard portions of the assembly should always be made with a strict project timeline in mind. Krasowski et $\mathrm{al}^{8}$ found that the pre-implementation stage for the shift to Beaker may encompass about two years. It may involve Subject Matter Experts' (SMEs) substantial effort, particularly with microbiology test and molecular biology test build and development of the usefulness of the Data Innovations Instrument Manager for middleware products. Substantially, we have noted that SME is a person who is an authority in a particular topic and these individuals play an integral role in the design, validation, and re-engineering sessions, which will take place during Epic implementation. For anatomical pathology laboratories preparing a switch to Beaker it is strongly advised, specifically for institutions not currently using Instrument Manager, that enough time, effort, and diligent know-how should be allocated to the specific topic of interfacing. Ultimately, another key decision point, which may need remarkable discussions where to build autoverification rules, but this area may be part of a subsequent critical review.

Overall, there are a few ways needed in implementing Beaker, as indicated by Powers. ${ }^{10}$ Beaker reports can permit pathology laboratories to operate faster and provide users with clear, effective and beneficial information for better decision-making. There is a personalizing Beaker application for each user. The new implementation allows data that streams into the system to swiftly flow right back out to clinicians and laboratories through operator-friendly dashboards. Currently, these dashboards and other reporting features are customized to different clients with different requirements. Beaker applications (such as the "Case Prep Worklist", the "Outstanding List", and the "Expected Receiving List") are customizable to display appropriate information for users. They are also replacing activity report panes within Beaker with custom-fit reports for the organization like "Result Entry".

The reporting view is able to deliver more of a whole picture view of a patient's health and, of course, patient's history for the anatomic and clinical pathologists. These physicians may require various views to make sure that at the end they are reaching the right clinical information, which is critically needed for the patient, to allow the pathologist to make the diagnosis considering all differential diagnoses. Retrieving histories means to include a gynecological history (eg, previous PAPs “ Papanicolaou tests”, microbiology data testing, and women's health data and history), patient's surgical and medical history (surgeries, medications, notes, social history, and upcoming appointments), or clinical history (with recent and historical complete blood counts and blood culture results). Each of these reports enables physicians to access appropriate information from within their subsequent roadmap. It is crucial to update regularly Chart Review to suit the requirements of each organization. This includes demonstrating suitable columns or adding custom print classes to the report view, improving satisfaction for providers and the laboratory. The Chart Review is intended to exhibit pertinent data in an easily usable and searchable layout. The report view is incredibly fundamental to how a customer interacts with Chart Review.

Moreover, enhancing front-facing tools and end product to better manage administration or long-term reporting is essential. Long-term reporting can be conducted in various circumstances varying on the time frame and data being demanded. Discovering the correct instrument for the knowledge requested is critical, and the reporting team should examine whether Clarity, Reporting Workbench, or SlicerDicer are the best means for the specific assignment at hand. Finally, developing custom dashboards to offer customers summaries is good. Dashboards are customizable by mission role and deliver an excellent platform for exhausting and summarizing reporting content. The views of dashboard can also vary depending on the role. For example, if customers have a crossover between laboratory and pathology, they may like different views to monitor information certain to their position. Reporting tools remain a solid measure to validate the impact on laboratory management and pathologist satisfaction, which 
is not to minimize. Empowering operators with valuable tools and functional platforms empowers them to partner with - instead of being dependent on - the Beaker IT team.

Another important aspect of academic centers is the implementation and exertion of EMR data for research. Milinovich and Kattan specifically focused on this aspect. ${ }^{11}$ For this specific data repository, they operate identifiers of the Unified Medical Language System (UMLS). The Meta-thesaurus from the UMLS blends tantamount terms and identical codes from distinct medical vocabularies into succinct phrases and identifiers. The subgroup can be packed into various database types. Some of the most classical databases are Microsoft SQL, MySQL, and Oracle. Streamlining the data structure into UMLS identifiers protects time in characterizing populations and isolating normalized data sets extremely useful for analysis. Normalizing data means to prepare data to be loaded into a structured database, which is labeled a data warehouse. Data warehouse stores massive amounts of data or big data in a structured format with the aim for ease of lookup. The database is constituted of predefined tables and columns that are determined by specific operational needs. During the normalization multiple processes take place with the purpose to scrub, reorganize, and reformat the data from different sources. Normalizing data means the elimination of duplicates, redundancies, inconsistencies, and anomalies with at the same time resolving data conflicts and maximizing data integrity, which is critical in any LIS. The absence of data normalization, raw data, means to deal with a jumble of inaccessible and potentially unusable elements. It is crucial to have the skill to streamline EMR research. Such a process has many advantages, particularly when dealing with enormous amounts of data. For example, at Cleveland Clinic, more than six and half million terms are plotted to UMLS identifiers. Such a process accounts for over $35 \times 10^{9}$ distinct data points in over $4 \times 10^{6}$ patients. Also, approximately $5 \times 10^{5}$ custom UMLS identifiers have been included at Cleveland Clinic to incorporate sites, providers, and their relationships with each other.

One of the greatest strengths of Beaker is end user versatility in the configurability function. In this sense, configurability has permitted to several organization to have fewer issues comparing with customization, which can harbor a risk when there are regular updates to the software. The potentiality to have a software, which is highly configurable, as Beaker is, the user is less prone to have difficulty with upgrades and updates. The "snapshot report" feature magnificently indicates the Beaker's configurability. In the snapshot report the end user can remain in an active window with the ability to read previous histories, surgical notes, proof consult notes, and screen clinical pathology results for a case. Dragon ${ }^{\mathrm{TM}}$ functionality, or similar speech recognition systems, have been useful for creating transcriptions in case results reports. With CoPath ${ }^{\mathrm{TM}}$, the pathologist uses a single-page Word document (Windows ${ }^{\mathrm{TM}}$ ) to make a pathology report, while with Epic Beaker the pathologists move between different fields, which may be cumbersome for some pathologists dealing with difficult specimens. In Table 1, a number of advantages and disadvantages are briefly listed. In many institutions, EPIC is powering their revolution into a single, cohesive, and integrated delivery system for health care. This incorporated system combines together all credentials tasks, including scheduling and registration, computerized provider order entry (CPOE), clinical charting, e-prescribing, and charge capture. In the United States of America and Canada, the Clinical Decision Support System (CDSS) is helping physicians to choose evidence-based decisions, regarding patients' needs and this is the major rational task, which is trying to be implemented worldwide.

In conclusion, Epic Beaker seems to be a good LIS, which may improve the workflow of laboratory diagnostic services. It is not devoid of disadvantages, but the versatility in the configurability of the user is paramount because upgrades and updates may merge smoothly with the pathologist's learning curve. Cost operability may be a challenge for some institutions, but the interaction between healthcare providers in the current world interconnected may characterize the popularity of this LIS. Finally, this report does not compare this product with other vendors and may not accurately evaluate and compare Epic Beaker with other benchmark products, which is the main limitation of this paper. Nevertheless and no matter which software we use, the world of the LIS and AP is continuously expanding at exponential speed. It is enormously improving in the laboratory diagnostics for a more efficient and effective service to both clinicians and patients. 
Table I Advantages and Disadvantages of EPIC Beaker

\begin{tabular}{|c|c|}
\hline Advantages & Disadvantages \\
\hline - Intuitive user interface with user friendly design and navigation & $\begin{array}{l}\text { - The learning curve from beginner to advanced user is not graciously } \\
\text { standardized because the learning modules may not be free accessible } \\
\text { to everybody }\end{array}$ \\
\hline - Clinicians can ensure that the specimen was received by the AP lab & $\begin{array}{l}\text { - It is difficult to correct mistakes or delete erroneously created } \\
\text { encounters or patient visits. }\end{array}$ \\
\hline $\begin{array}{l}\text { - Continuing updated technology and features with consistent upgrade } \\
\text { cadence and breadth of capabilities. }\end{array}$ & $\begin{array}{l}\text { - It is often difficult to create a letter or a work excuse on a hospital } \\
\text { template. }\end{array}$ \\
\hline - Discrete fields to enter test results & $\begin{array}{l}\text { - It is difficult to evaluate patient information across platforms or other } \\
\text { operating systems }\end{array}$ \\
\hline $\begin{array}{l}\text { - Speed and accuracy are reached using shortcuts, templates, and smart } \\
\text { phrases }\end{array}$ & $\begin{array}{l}\text { - Visual updates can be worrisome and need to have a better flow in } \\
\text { explaining them to the users }\end{array}$ \\
\hline $\begin{array}{l}\text { - Templates include prepopulated fields, such as synoptic reports of the } \\
\text { College of the American Pathologists }\end{array}$ & $\begin{array}{l}\text { - Costly implementation and steepness of the learning curve may } \\
\text { dependent to the level of the education of the user }\end{array}$ \\
\hline - Ability to perform batch reporting for high-volume tests & - Dedicated support staff may appear limited to the user \\
\hline \multicolumn{2}{|l|}{ - Ability to track workflow within Beaker for easy access by directors } \\
\hline \multicolumn{2}{|l|}{ - Consistency between screens } \\
\hline \multicolumn{2}{|l|}{ - Decreased number of Excel ${ }^{\mathrm{TM}}$ spreadsheets used to track items } \\
\hline \multicolumn{2}{|l|}{$\begin{array}{l}\text { - The request of special stains, immunohistochemical orders, or } \\
\text { molecular biology orders is fast and accurate so that the pathologist } \\
\text { does not have to call the technologist }\end{array}$} \\
\hline \multicolumn{2}{|l|}{$\begin{array}{l}\text { - Ability to have a PDMP link with the precious function to search for } \\
\text { what controlled medications (eg, opiates) patients have been prescribed } \\
\text { with critical relevance in forensic cases originated at healthcare } \\
\text { institutions. }\end{array}$} \\
\hline \multicolumn{2}{|l|}{$\begin{array}{l}\text { - Interdisciplinary Communication is paramount and the entire care } \\
\text { team individuals can communicate with each other making decisions and } \\
\text { patient safety remarkably improved by seeing past decision making } \\
\text { processes. }\end{array}$} \\
\hline \multicolumn{2}{|l|}{$\begin{array}{l}\text { - Secure Chat feature in which HIPAA supported messages can be sent } \\
\text { to other institution's employee with the patient's name and record } \\
\text { attached. }\end{array}$} \\
\hline $\begin{array}{l}\text { - Patient Communication: Epic Beaker links to MyChart }{ }^{\mathrm{TM}} \text {, which is a } \\
\text { platform via which patients can search their own information without } \\
\text { the need to call the physicians. }\end{array}$ & \\
\hline
\end{tabular}

Notes: AP, anatomic pathology; "batch reporting" is the submission of multiple reports, or corrections to reports, in batch files that are formatted following detailed specifications; HIPAA, Health Insurance Portability and Accountability Act, an United States legislation that provides data privacy and security provisions regarding medical information; PDMP, prescription drug monitoring program. Although we tried to comprehensively evaluate the system and give a frank opinion, some of the issues may have been solved or are currently being tackled by the company.

\section{Disclosure}

The author is not affiliated with Epic Systems Corporation, but this software was implemented at the University Alberta Hospital in 2019. The author has neither direct or indirect stocks involved with this corporation. The author receives royalties from Springer and Nova publishers but these royalties go to pediatric charities. The author has nothing else to disclose. 


\section{References}

1. Sergi C, Davis DD. Incident Reporting. Treasure Island (FL): StatPearls; 2020.

2. Sergi C, Mikuz G. External quality assurance as a revalidation method for pathologists in pediatric histopathology: comparison of four international programs. BMC Clin Pathol. 2008;8:11.

3. Solez K, Bernier A, Crichton J, et al. Bridging the gap between the technological singularity and mainstream medicine: highlighting a course on technology and the future of medicine. Glob J Health Sci. 2013;5(6):112-125.

4. Sergi CM. Digital Pathology: the time is now to bridge the gap between medicine and technological singularity. In: Cvetković D, editor. Interactive Multimedia - Multimedia Production and Digital Storytelling. London, United Kingdom: IntechOpen; 2019.

5. Scott GD, Schrandt C, Ho CC, Chung MC, Zhou D, Shi RZ. Interfacing Complex Laboratory Instruments during a Change to Epic Beaker. $J$ Pathol Inform. 2018;9:24.

6. Blau JL, Wilford JD, Dane SK, et al. Implementation of epic beaker anatomic pathology at an academic medical center. J Pathol Inform. $2017 ; 8: 47$.

7. Chung MC, Gombar S, Shi RZ. Implementation of automated calculation of free and bioavailable testosterone in epic beaker laboratory information system. J Pathol Inform. 2017;8:28.

8. Krasowski MD, Wilford JD, Howard W, et al. Implementation of Epic Beaker Clinical Pathology at an academic medical center. J Pathol Inform. 2016;7:7.

9. VanSandt M, Turner K, Dash R, et al. Pathologist Opinions about EPIC Beaker AP: a Multi-Institutional Survey of Early Adopters. $J$ Med Syst. 2020;44(6): 111.

10. Powers M. 5 ways to boost lab efficiency and improve patient care through Epic's Beaker LIS reports. Available from: https://www.nordicglobal. com/blog/5-ways-to-boost-lab-efficiency-and-improve-patient-care-through-epics-beaker-lis-reports. Accessed February 16, 2022.

11. Milinovich A, Kattan MW. Extracting and utilizing electronic health data from Epic for research. Ann Transl Med. 2018;6(3):42.

Risk Management and Healthcare Policy

\section{Publish your work in this journal}

Risk Management and Healthcare Policy is an international, peer-reviewed, open access journal focusing on all aspects of public health, policy, and preventative measures to promote good health and improve morbidity and mortality in the population. The journal welcomes submitted papers covering original research, basic science, clinical \& epidemiological studies, reviews and evaluations, guidelines, expert opinion and commentary, case reports and extended reports. The manuscript management system is completely online and includes a very quick and fair peer-review system, which is all easy to use. Visit http://www.dovepress.com/testimonials.php to read real quotes from published authors.

Submit your manuscript here: https://www.dovepress.com/risk-management-and-healthcare-policy-journal 